\title{
Germanium quantum well with two subbands occupied: kinetic properties
}

\author{
I.B. Berkutov ${ }^{1,2,3}$, V.V. Andrievskii ${ }^{1,2}$, Yu.F. Komnik ${ }^{1}$, and O.A. Mironov²,4 \\ ${ }^{1}$ B. Verkin Institute for Low Temperature Physics and Engineering of the National Academy of Sciences of Ukraine \\ 47 Nauky Ave., Kharkiv 61103, Ukraine \\ E-mail: Andrievskii@ilt.kharkov.ua \\ ${ }^{2}$ Institute of Low Temperature and Structure Research, Polish Academy of Sciences \\ ul. Okólna 2, 50-422, Wrocław, Poland \\ ${ }^{3}$ Department of Physics, North Carolina State University, Raleigh, NC 27695, USA \\ ${ }^{4}$ Department of Physics, University of Warwick, Coventry CV4 7AL, UK
}

Received February 7, 2017, published online August 27, 2017

\begin{abstract}
Multisubband transport of charge carriers in the $p$-type $\mathrm{Si}_{0.4} \mathrm{Ge}_{0.6} / \mathrm{Ge} / \mathrm{Si}_{0.4} \mathrm{Ge}_{0.6}$ heterostructure has been investigated by means of magnetotransport measurements at low temperatures and high magnetic fields. Two frequency Shubnikov-de Haas oscillations indicate occupation of two subbands. This allows us to determine the densities and mobilities of the charge carriers on each subband. Shubnikov-de Haas oscillations reveal two 2D conduction subbands with carrier effective masses of $0.112 m_{0}$ and $0.131 m_{0}$. The quantum Hall ferromagnetic states which results from the crossing of two Landau levels with opposite spin belongings to different subband was observed in SiGe systems for the first time.
\end{abstract}

PACS: 72.20. My Galvanomagnetic and other magnetotransport effects;

73.20.Fz Weak or Anderson localization.

Keywords: semiconductor heterostructure, magnetotransport, Shubnikov-de Haas oscillations.

\section{Introduction}

Usage the semiconductor heterostructures with two-dimensional (2D) electron gas as the functional materials for nanoelectronics requires knowledge of basic characteristic parameters of mobile charge carriers such as effective mass, density and mobility, as well as information about the interaction between them and other quasiparticles. This problem can be solved by study of the behavior of their conductivity at different temperatures and magnetic fields. Especially valuable are the investigations of low-temperature Shubnikov-de Haas (SdH) oscillations of magnetoresistance. SdH oscillations measurements are often employed to characterize the properties of 2D electron gas at semiconductor heterointerfaces. Quantum oscillations in the transport properties of metallic and semiconducting materials arise due to the formation of magnetic quantum levels in spectrum (Landau levels), which allows to determine the energy of the conduction electrons and other parameters. They arise directly from the magnetic field dependence of the conduction electron energies, and are, therefore, a powerful experimental probe of the electronic bandstructure close to the Fermi energy [1]. They also provide band-specific details of conduction electron properties such as effective mass and mobility.

The methods of the experimental data processing for two-dimensional systems in heterostructures in the case of a single subband occupied are well-known. The problem of the calculations of mobile charge carriers parameters becomes more complicated in the case of two subbands occupied. In the case of the two subbands occupation the problem of the mobile charge carriers parameters determination become much more complicated because the process of electron transport involves two groups of carriers and it is necessary to obtain information about the kinetic parameters of each of these groups, while the obtained experimental data related to the sample as a whole. In the case of multisubband occupation, coupling among the dis- 
tribution functions of the electrons in different subbands arises due to intersubband scattering, which should contribute to the total magnetoconductivity.

In this paper the kinetic properties of the charge carriers in $p$-type SiGe heterostructure with pure germanium quantum well and two subbands occupied are study. Also the phenomenon of the unpolarized ferromagnetic transitions in quantum Hall ferromagnetic state is shown.

\section{Sample structure}

The sample under study is $p$-type $\mathrm{Si}_{0.4} \mathrm{Ge}_{0.6} / \mathrm{Ge} / \mathrm{Si}_{0.4} \mathrm{Ge}_{0.6}$ heterostructure with fully strained germanium $19.6 \mathrm{~nm}$ wide quantum well. It was produced using a hybrid-epitaxy methodology utilizing ultra high vacuum chemical vapour deposition (UHV-CVD) for the virtual substrate and solid source molecular beam epitaxy (SS-MBE) for the strained active layers [2]. The virtual substrate consisted of $6 \mu \mathrm{m}$ $\mathrm{Si}_{1-y} \mathrm{Ge}_{y}$ capped with $1 \mu \mathrm{m}$ layer of constant composition $\mathrm{Si}_{0.4} \mathrm{Ge}_{0.6}$. A high temperature $\left(860{ }^{\circ} \mathrm{C}\right)$ desorb was performed in the SS-MBE chamber prior to re-growth of an initial $200 \mathrm{~nm}$ lattice-matched $\mathrm{Si}_{0.4} \mathrm{Ge}_{0.6}$ layer at $800{ }^{\circ} \mathrm{C}$ to ensure good crystalline quality. Growth was interrupted while the substrate cooled to $350{ }^{\circ} \mathrm{C}$ for growth of the active layers. The heterostructure consisted of three relaxed $\mathrm{Si}_{0.4} \mathrm{Ge}_{0.6}$ layers: $20 \mathrm{~nm}$ uniform layer, $5 \mathrm{~nm}$ supply layer boron doped at $2 \cdot 10^{18} \mathrm{~cm}^{-3}$ and $15 \mathrm{~nm}$ set back layer, followed by the strained Ge channel, $20 \mathrm{~nm} \mathrm{Si}_{0.4} \mathrm{Ge}_{0.6}$ capping layer and $2 \mathrm{~nm}$ of Si surface termination. We should point out that the quantum well was prepared as "pure Ge".
A number of analytical techniques (uleSIMS, HRXRD and RBS) have been used to characterize the matrix content, layer thicknesses and level of strain in the active channel. These all show the samples have high crystallinity, but the channel in fact contained a small amount (2-5\%) of Si [3]. Sample employed buried layer doping as the lower interface of the Ge channel is smoother, which leads to a reduction of interface scattering effects, which would hinder the mobility. The conducting region had Hall-bar configuration in the form of a narrow strip $0.05 \mathrm{~mm}$ wide and with a distance between the two pairs of narrow potential leads $0.5 \mathrm{~mm}$. The measurements were performed by standard lock-in methodic at temperatures $1.5-100 \mathrm{~K}$ in magnetic fields up to $14 \mathrm{~T}$.

\section{The kinetic characteristics calculations}

The experimental dependences of resistance $\rho_{x X}(B)$ vs. magnetic field are shown in Fig. 1(a). The analysis of those dependences using fast Fourier transformation (FFT) revealed two maxima (see Fig. 1(b)) and it suggests (together with the observed beats of the Shubnikov-de Haas oscillations) that there are two subbands occupied in the sample under study. The maxima of Fourier spectrum with frequencies $f_{1}$ and $f_{2}$ represent lower and upper subbands, respectively. Values of the charge carrier densities on each subbands were obtained using the relationship $p_{i}=2 e f_{i} / h$. The values of the calculated densities are shown in Table 1.

In two-dimensional conducting systems in case of two subbands occupied the density $p_{H}$ and mobility $\mu_{H}$, found by Hall effect study, can be represented as $[4,5]$

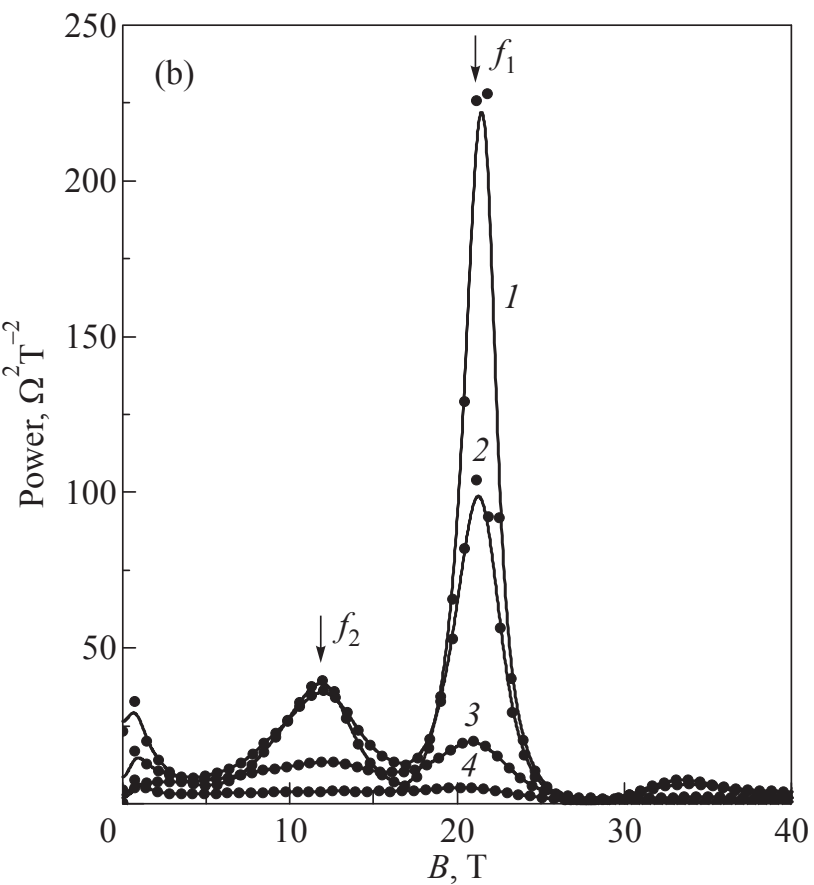

Fig. 1. The magnetic field dependences of sample resistance (a) and FFT spectrum of SdH oscillations (b) at different temperatures, K: 1.45 (1), 4.5 (2), 10 (3), 15 (4). 
Table 1. The kinetic characteristics of the charge carriers calculated from Hall measurements and the characteristics of the charge carriers on each subband

\begin{tabular}{c|c|c|c|c|c|c|c|c}
\hline \hline \multicolumn{3}{c|}{ Sample characteristics } & \multicolumn{6}{c}{ Subbands characteristics } \\
\hline$p_{H,} \mathrm{~m}^{-2}$ & $\mu_{H}, \mathrm{~m}^{2} /(\mathrm{V} \cdot \mathrm{c})$ & $E_{F}, \mathrm{meV}$ & $i$ & $p_{i}, \mathrm{~m}^{-2}$ & $\mu_{i}, \mathrm{~m}^{2} /(\mathrm{V} \cdot \mathrm{c})$ & $E_{i}, \mathrm{meV}$ & $\alpha_{i}$ & $m^{*}, m_{0}$ \\
\hline \multirow{2}{*}{$1.62 \cdot 10^{16}$} & \multirow{2}{*}{2.4} & \multirow{2}{*}{33.279} & 1 & $1.06 \cdot 10^{16}$ & 2.665 & 12.545 & 6.2 & 0.112 \\
& & & 2 & $6.09 \cdot 10^{15}$ & 1.845 & 32.624 & 18 & 0.131 \\
\hline \hline
\end{tabular}

$$
\begin{gathered}
p_{H}=\frac{\left(p_{1} \mu_{1}+p_{2} \mu_{2}\right)^{2}}{p_{1} \mu_{1}^{2}+p_{2} \mu_{2}^{2}}, \\
\mu_{H}=\frac{p_{1} \mu_{1}^{2}+p_{2} \mu_{2}^{2}}{p_{1} \mu_{1}+p_{2} \mu_{2}},
\end{gathered}
$$
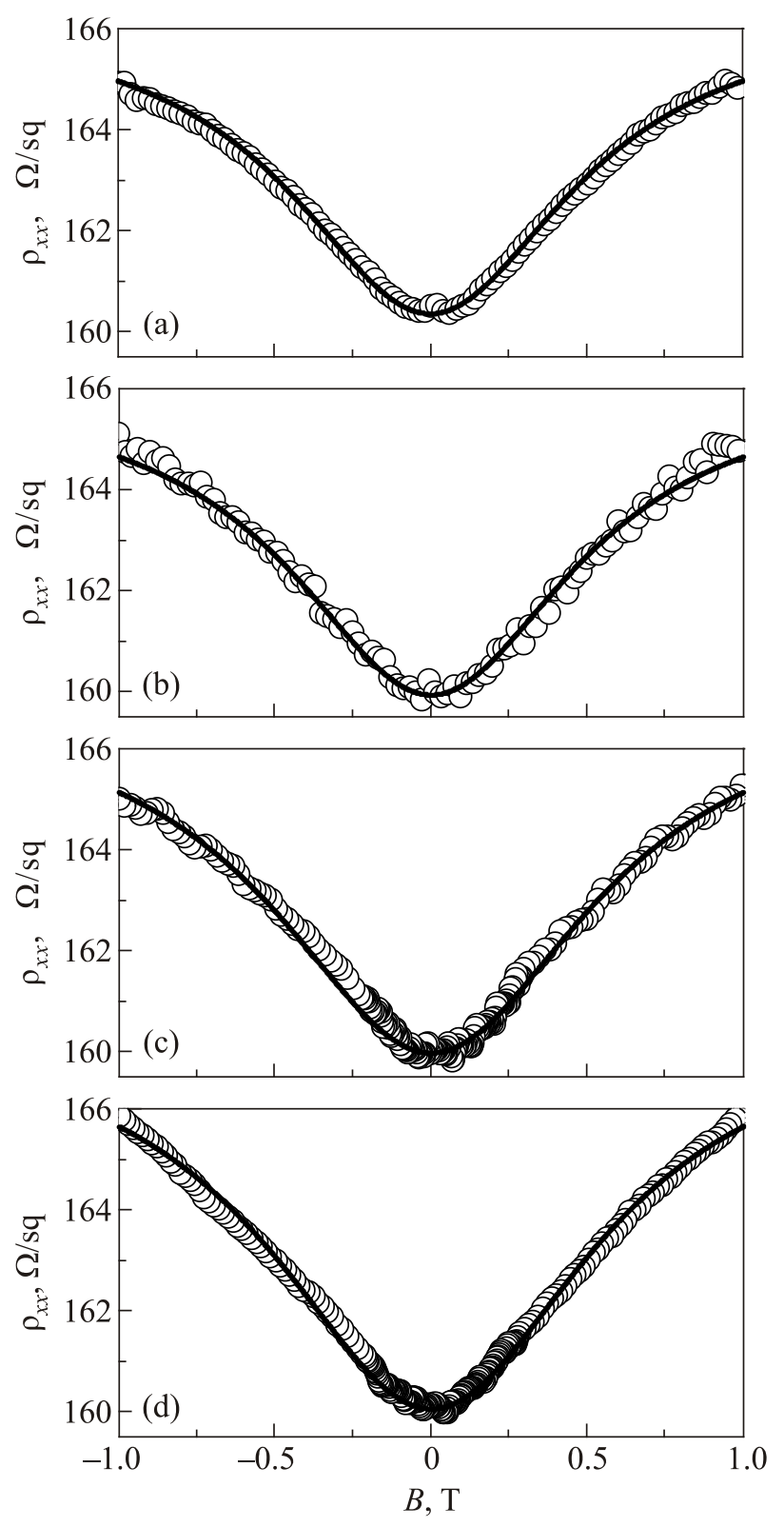

Fig. 2. The magnetic field dependences of sample resistance $\rho_{X X}(B)$ at temperatures, K: 1.45 (a), 3.5 (b), 5.5 (c), 10 (d). Solid lines are dependences calculated according to Eq. (3). here $p_{1}$ and $p_{2}$ are the densities, $\mu_{1}$ and $\mu_{2}$ are the mobilities of charge carriers on the corresponding subbands. Mobility values found from simultaneous solution of Eqs. (1) and (2) are shown in Table 1.

According to the semi-classical approximation in the two-dimensional conducting system in the presence of perpendicular magnetic field, there are two groups of charge carriers with the densities $p_{i}$ and mobilities $\mu_{i}$ which participate in the transfer of charge independently. Last statement is not true in real 2D systems in the general case, and the inter-subband interaction can be quite substantial. This fact was taken into account in the theory [6] in which the expression for the magnetoresistance in the semi-classical approximation was obtained as follows:

$$
\rho_{x x}(B)=\rho_{0}\left(1+\frac{r p_{1} p_{2} \mu_{1} \mu_{2}\left(\mu_{1}-\mu_{2}\right)^{2} B}{\left(p_{1} \mu_{1}+p_{2} \mu_{2}\right)^{2}+\left(r p_{H} \mu_{1} \mu_{2}\right)^{2} B^{2}}\right),
$$

where $\rho_{0}=1 /\left(p_{1} \mu_{1}+p_{2} \mu_{2}\right) e$ is the zero magnetic field resistance, $r$ is the dimensionless parameter which characterizes interlayer interaction. When $r=1$, Eq. (3) goes to the usual expression for noninteracting conducting channels. This model describes positive magnetoresistance which saturates when both groups of carriers reach conditions of semi-classical strong magnetic field at $\mu_{i} B \gg 1$. When the mobilities on both subbands are absolutely equal, this theory not can be applied. Examples of description of the experimental magnetoresistance dependences of the sample under study, performed according to Eq. (3), shown in Fig. 2. It gives possibility to calculate the value $r$. It was found that this parameter is slightly changes from 0.92 to 0.8 in the temperature range $1.45-10 \mathrm{~K}$. It means that there are two weakly interacting systems of charge carriers which occupied two subbands.

\section{The effective mass calculations}

The effective mass $m^{*}$ and quantum scattering time $\tau_{q}$ are usually estimated from temperature and magnetic field dependences of the SdH oscillation amplitudes. The change in the conductivity of the $2 \mathrm{D}$ gas in a region of essential quantum effects was considered theoretically in Ref. 7:

$\rho_{x X}=\frac{1}{\sigma_{0}}\left[1+4 \sum_{s=1}^{\infty}\left(\frac{\Psi s}{\operatorname{sh} \Psi s}\right) \exp \left(-\frac{\pi s}{\omega_{c} \tau_{q}}\right) \cos \left(\frac{2 \pi s \varepsilon_{F}}{\hbar \omega_{c}}-\Phi\right)\right]$, 
where $\Psi=2 \pi^{2} k_{B} T /\left(\hbar \omega_{c}\right)$ determines the temperature and magnetic field dependences of the oscillations amplitude, $\omega_{c}=e B / m^{*}$ is the cyclotron frequency, $\tau_{q}$ is the quantum (single particle) relaxation time of charge carriers, which describes homogeneous broadening of Landau levels, $\Phi$ is the phase. The Fermi energy is $\varepsilon_{F}=\pi \hbar^{2} n / m^{*}$ in the 2D case ( $n$ is the density of charge carriers). Practically the term of the sum with $s=1$ is sufficient in Eq. (1). The first term describes a damping of the $\mathrm{SdH}$ oscillations due to the temperature broadening of the Fermi function. The method of effective mass $m^{*}$ and quantum scattering time $\tau_{q}$ determination is described in Ref. 8 , but as shown therein, it is not applicable in case of two subband occupied.

The effective mass of charge carriers can be estimated from the temperature decay of the FFT peaks in the power spectrum. At the given a magnetic field interval, one can numerically construct $\rho_{X X}(B)$ from the formula (4) and Fourier transforms in order to compare the peak height with the measured data. The temperature dependence of the FFT maxima should follow same dependence:

$$
A_{F F T} \sim \frac{\Psi}{\operatorname{sh} \Psi} .
$$

The zero-field resistivity and the hole density are read from the experimental data while $m^{*}$ and $\tau_{q}$ are fitting parameters. To provide robustness to the procedure, the fit is performed on the amplitude of a peak as a function of temperature. As it seen from Fig. 1(b), the highs of Fourier spectrum maxima with frequencies $f_{1}$ and $f_{2}$ have different temperature dependences which means that the effective masses of charge carriers at first and second subbands can be different as found in Ref. 3. The results of such calculation are shown in Fig. 3. The two different values of

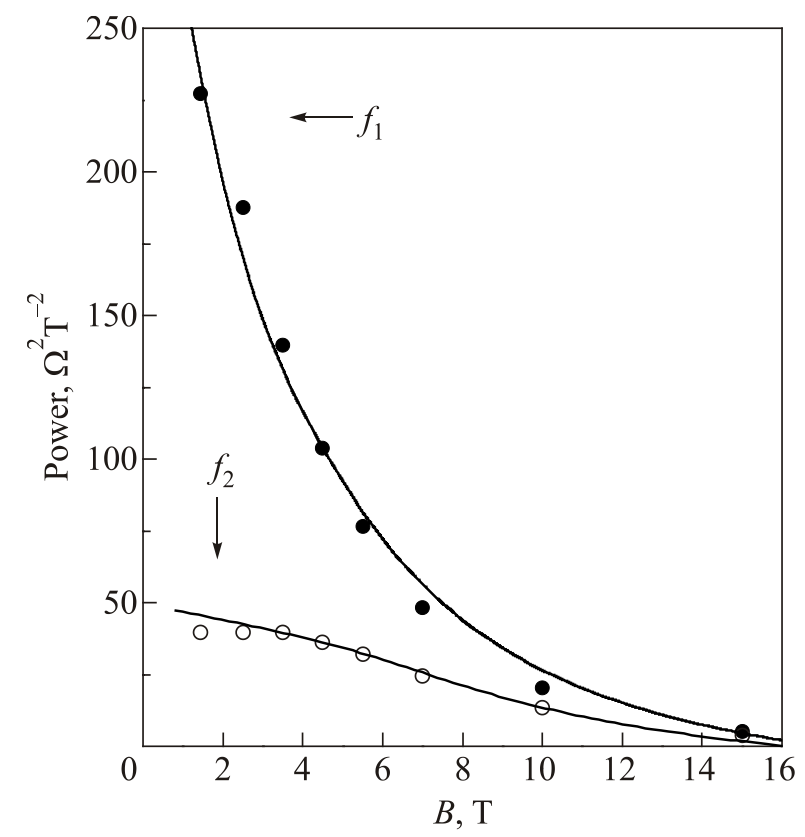

Fig. 3. Peaks heights of harmonics $f_{1}(\bullet)$ and $f_{2}(\bigcirc)$ as a function of temperature together with a fit (5). the effective mass $m_{1}^{*}=0.118 m_{0}, m_{2}^{*}=0.131 m_{0}$ and two values of parameter $\alpha=\tau / \tau_{q}$ such as $\alpha_{1}=4.5, \alpha_{2}=14$ on first and second subbands were estimated ( $\tau$ is the transport scattering time of charge carries).

Calculation of the effective mass value of the sample under study was carried out according to the theoretical model $[9,10]$ using the values of $p_{1}, p_{2}, \mu_{1}$ and $\mu_{2}$ found above. In sufficiently weak magnetic fields when the number of filled Landau levels is large, the resistance of twodimensional systems can be represented as

$$
\rho=\rho^{(0)}+\rho^{(1)}+\rho^{(2)},
$$

where $\rho^{(0)}$ is the classical resistance, $\rho^{(1)}$ is the first-order quantum correction describing the SdH oscillations, $\rho^{(2)}$ is the second-order quantum contribution. According to the theory $[9,10]$ the classical resistance is

$$
\rho^{(0)}=\frac{m^{*}}{e^{2} p_{s}} \frac{\omega_{c}^{2} v_{s}+v_{0} v_{r}^{2}}{\omega_{c}^{2}+v_{0}^{2}},
$$

here $p_{s}=p_{1}+p_{2}$ is the total density of charge carriers, $\omega_{c}$ is the cyclotron frequency. The characteristic rates $v_{s}, v_{r}$ and $v_{0}$ are given by

$$
\begin{gathered}
v_{s}=\left(\frac{p_{1}}{p_{s}}\right) v_{11}^{\mathrm{tr}}+\left(\frac{p_{2}}{p_{s}}\right) v_{22}^{\mathrm{tr}}+v_{12}^{\mathrm{tr}}, \\
v_{r}=\left(\frac{p_{2}}{p_{s}}\right) v_{11}^{\mathrm{tr}}+\left(\frac{p_{1}}{p_{s}}\right) v_{22}^{\mathrm{tr}}+2 v_{12}-v_{12}^{\mathrm{tr}}, \\
v_{0}=\frac{d}{v_{r}}, d=\left(v_{11}^{\mathrm{tr}}+v_{12}\right)\left(v_{22}^{\mathrm{tr}}+v_{12}\right)-\frac{\left(v_{12}-v_{12}^{\mathrm{tr}}\right)^{2} p_{s}^{2}}{4 p_{1} p_{2}} .
\end{gathered}
$$

The first-order quantum contribution is

$$
\begin{aligned}
\rho^{(1)} & =-\sum_{j=1,2} I_{j} \frac{2 m_{j}^{*}}{e^{2} p_{s}}\left[\frac{2 p_{j}}{p_{s}} v_{j j}^{\mathrm{tr}}+v_{12}^{\mathrm{tr}}\right] \times \\
& \times \exp \left(-\alpha_{j}\right) \cos \frac{2 \pi\left(E_{F}-E_{j}\right)}{\hbar \omega_{c j}},
\end{aligned}
$$

$\alpha_{j}=\pi v_{j} / \omega_{c j}, v_{j}=v_{j j}+v_{12}, E_{j}$ are the subband energies, $I_{j}=X_{j} / \operatorname{sh} X_{j}$, where $X_{j}=2 \pi T / \hbar \omega_{c j}$.

The second order quantum contribution is given by

$$
\begin{aligned}
\rho^{(2)}= & \frac{2 m_{1}^{*} p_{1}}{e^{2} p_{s}^{2}} v_{11}^{\mathrm{tr}} \mathrm{e}^{-2 \alpha_{1}}+\frac{2 m_{2}^{*} p_{2}}{e^{2} p_{s}^{2}} v_{22}^{\mathrm{tr}} \mathrm{e}^{-2 \alpha_{2}}+ \\
& +\frac{2 \bar{m}^{*}}{e^{2} p_{s}} v_{12}^{\mathrm{tr}} \mathrm{e}^{-\alpha_{1}-\alpha_{2}} \cos \frac{2 \pi \Delta_{12}}{\hbar \bar{\omega}_{c}},
\end{aligned}
$$

where $\Delta_{12}=E_{2}-E_{1}$.

The system of equations (7), (8) was solved numerically and it gives possibility to describe the experimental magnetoresistance data at different temperatures. The example 


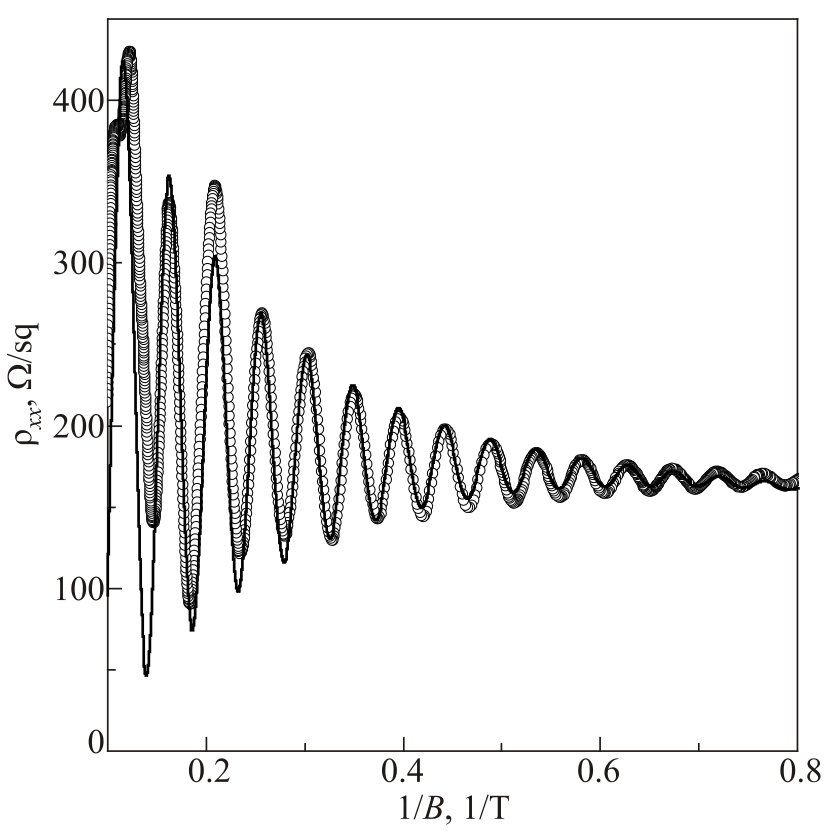

Fig. 4. The dependence of resistivity $\rho_{x x}$ is plotted against the inverse magnetic field at temperature $1.45 \mathrm{~K}$. Solid line is dependence calculated according to Eqs. (7)-(9).

of the oscillation part of magnetoresistance description is shown in Fig. 4. In the Table 1 the values of effective mass $m^{*}$, Fermi energy $E_{F}$, the energies of each subband $E_{1}$ and $E_{2}$, parameters $\alpha_{1}$ and $\alpha_{2}$ are shown. Two different values of the effective mass and two different values of parameter $\alpha$ were found on first and second subband, respective- ly: $m_{1}^{*}=0.12 m_{0}, \alpha_{1}=6.2$ and $m_{2}^{*}=0.147 m_{0}, \alpha_{2}=18$, which are close to the values estimated before.

\section{Manifestations of the ferromagnetic state}

Magnetic field dependence of the diagonal and offdiagonal (Hall) components of the resistance is shown in Fig. 5. It should be noted, that there is unusual feature 1 (in the circled area) observed at magnetic fields $B \sim 9 \mathrm{~T}$. In this area the differential Hall resistance $d R_{x y} / d B$ can be negative during the $B$ scan. This behavior is certainly unusual. In a one-band system only positive differential Hall resistance (i.e., the classical Hall resistance or in the region between two plateaus) and zero differential Hall resistance (i.e., in the plateau region) have been observed. The similar features were studied earlier in $n$-type GaAs/AlGaAs quantum well $[11,12]$. It was argued that ferromagnetic quantum Hall states, due to the crossing of two Landau levels with opposite spin and different subband indiced, are responsible for these unusual feature.

When the two sets of spin-split Landau levels are far away from each other (regime I, Fig. 5), then both spin states of the first subband are filled like an ordinary, unpolarized quantum Hall state. When two Landau levels with opposite spins and different subbands approach each other (regime II, Fig. 5), there is now an opportunity for the electrons in the first subband to occupy the up-spin state of the second subband. By spin-flipping to the second subband, the electrons can actually save exchange energy. This phenomenon can be interpreted as a transition from
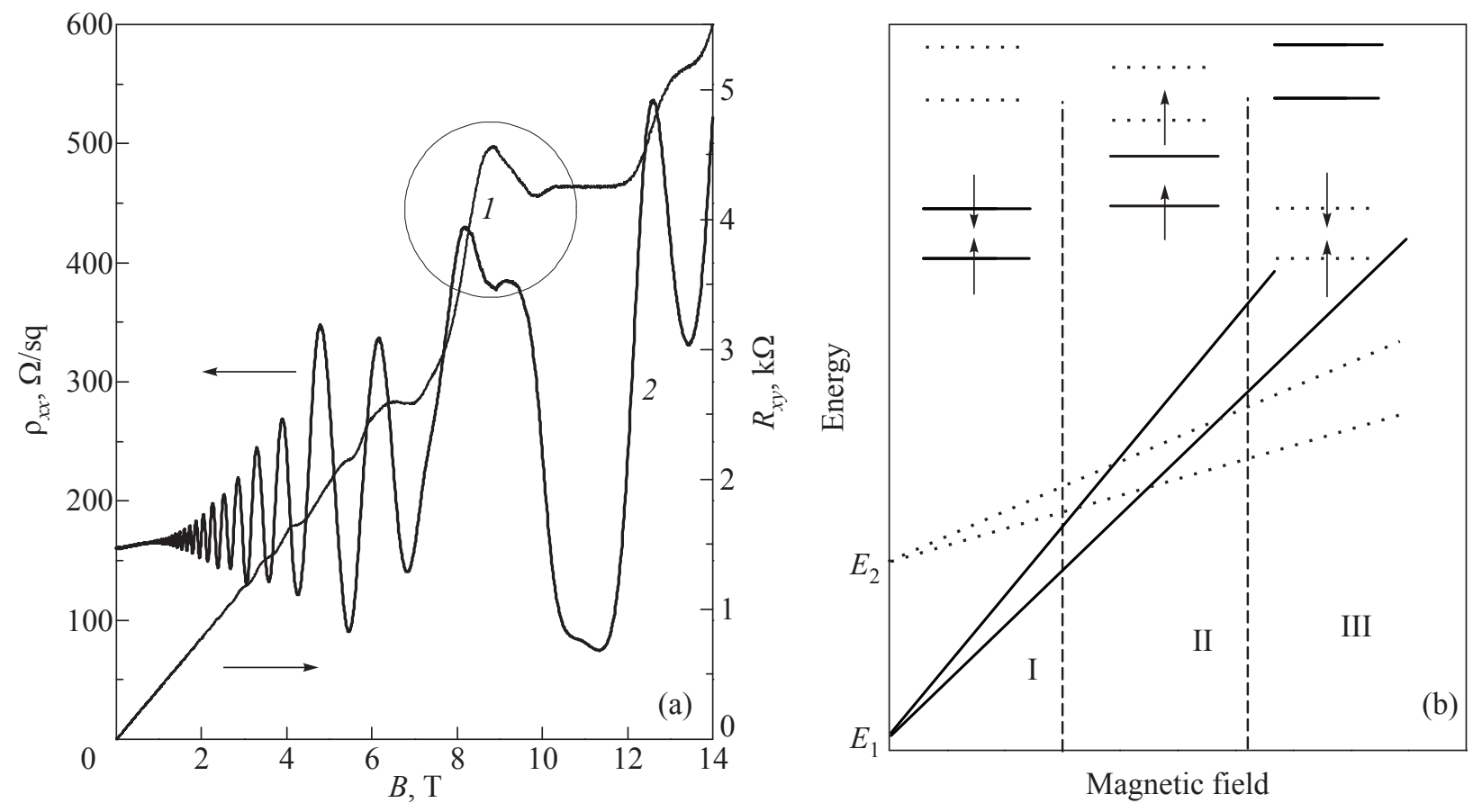

Fig. 5. Magnetic field dependences of the diagonal component $\rho_{x x}(B)$ and off-diagonal (Hall) component $R_{x y}(B)$ of the resistance (a). Energy diagram of two sets of Landau levels that are brought together by the magnetic field in condition of the ferromagnetic state transition (b). 


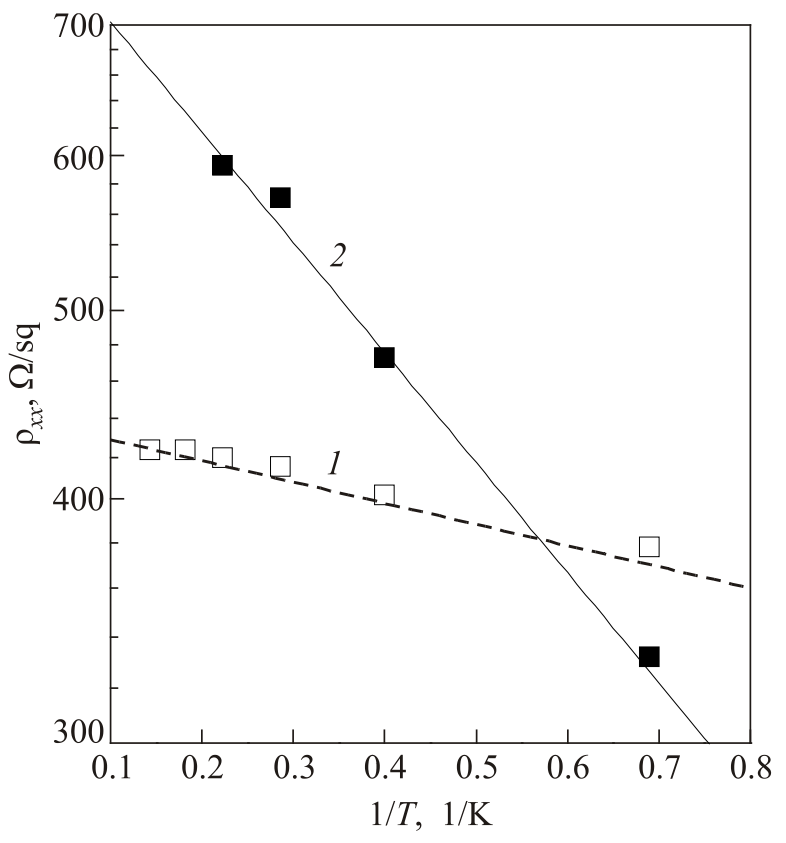

Fig. 6. The longitudinal resistivity $\rho_{x x}$ is plotted against the inverse temperature in the Arrehenius plots for points 1 (open squares) and 2 (solid squares) in Fig. 5. The straight lines are linear regression of experimental data according to Arrhenius equation with activation energies $0.5 \mathrm{~K}(1)$, $2.6 \mathrm{~K}$ (2).

a paramagnetic to ferromagnetic state. The phase transition is expected to take place when the energy spacing between two Landau levels is roughly equal to the exchange energy. So the quantum Hall state in this regime is a ferromagnetic state. Likewise, at the higher $B$ side, when the energy spacing becomes larger than the exchange energy (regime III, Fig. 5), there is another transition back to conventional unpolarized quantum Hall states where two spin states of the upper subband are occupied. The dependences of resistance $\rho_{x x}$ as a function of reversed temperature taken from 1 and 2 in Fig. 5 are shown in Fig. 6 (open and solid squares, respectively). The calculations using Arrhenius equation reveal that energies in the regions 1 and 2 are 0.5 and $2.6 \mathrm{~K}$, respectively, which is evidence that the activation energy in ferromagnetic state is significantly smaller than the energy spacing between two Landau levels.

\section{Conclusion}

In conclusion, we performed detailed experimental investigations of the magnetotransport in the $p$-type heterostructure $\mathrm{Si}_{0.4} \mathrm{Ge}_{0.6} / \mathrm{Ge} / \mathrm{Si}_{0.4} \mathrm{Ge}_{0.6}$ with two occupied subbands in Ge channel. This evidence was caused both facts: the SdH oscillation observations, which demonstrate a "beating” phe- nomena and Fourier analysis. The methodology of charge carriers kinetic characteristics calculation such as hole densities and mobilities on each from subbands is represented. The hole densities of the occupied subbands are: $1.06 \cdot 10^{16} \mathrm{~m}^{-2}$ (1st subband) and $6.09 \cdot 10^{15} \mathrm{~m}^{-2}$ (2nd subband), with motilities 2.665 and $1.845 \mathrm{~m}^{2} /(\mathrm{V} \cdot \mathrm{c})$, respectively. The numerical calculations of effective mass received the values $m^{*}=0.112 m_{0}$ (for the 1 st subband) and $m^{*}=0.131 m_{0}$ (for the 2 nd subband). The evidence of manifestation of a ferromagnetic state in the magnetic field $\sim 9 \mathrm{~T}$ is found. This state appears when Landau levels with different subband and orbital induces crossing at the Fermi level act as up and down pseudospin levels. The corresponding value of activation energy was equal $0.5 \mathrm{~K}$.

\section{Acknowledgments}

The authors are grateful to O.E. Raichev for useful discussions. Measurements were made in Institute of Low Temperature and Structure Research, Polish Academy of Sciences.

1. D. Shoenberg, Magnetic Oscillations in Metals, Cambridge University Press, Cambridge (1984).

2. R.J.H. Morris, T.J. Grasby, R. Hammond, M. Myronov, O.A. Mironov, D.R. Leadley, T.E. Whall, E.H.C. Parker, M.T. Currie, C.W. Leitz, and E.A. Fitzgerald, Semicond. Sci. Technol. 19, L106 (2004).

3. O.A. Mironov, M. Goiran, J. Galibert, D.V. Kozlov, A.V. Ikonnikov, K.E. Spirin, V.I. Gavrilenko, G. Isella, M. Kummer, H. von Känel, O. Drachenko, M. Helm, J. Wosnitza, R.J.H. Morris, and D.R. Leadley, J. Low Temp. Phys. 159, 216 (2010).

4. S. Mori and T. Ando, Phys. Rev. B 19, 6433 (1979).

5. H.L. Stormer, A.C. Gossard, and W. Weigmann, Solid State Commun. 43, 707 (1982).

6. E. Zaremba, Phys. Rev. B 45, 14143 (1992).

7. A. Isihara and L. Smrčka, J. Phys. C 19, 6777 (1986).

8. I.B. Berkutov, V.V. Andrievskii, Yu.F. Komnik, Yu.A. Kolesnichenko, R.J.H. Morris, D.R. Leadley, and O.A. Mironov, Fiz. Nizk. Temp. 38, 1455 (2012) [Low Temp. Phys. 38, 1145 (2012)].

9. O.E. Raichev, Phys. Rev. B 78, 125304 (2008).

10. N.C. Mamami, G.M. Gusev, E.C.F. da Silva, O.E. Raichev, A.A. Quivy, and A.K. Bakarov, Phys. Rev. B 80, 085304 (2009).

11. X.Y. Lee, H.W. Jiang, and W.J. Schiaff, Phys. Rev. Lett. 83, 3701 (1999).

12. X.C. Zhang, D.R. Faulhaber, and H.W. Jiang, Phys. Rev. Lett. 95, 216801 (2005). 